OPEN A Functional p53 Responsive

SUBJECT AREAS:

RISK FACTORS

BREAST CANCER

Received

13 May 2014

Accepted

27 August 2014

Published

15 September 2014

Correspondence and requests for materials should be addressed to

X.N. (niexiuyishi@

126.com) or X.P.M.

(miaoxp@mail.hust. edu.cn)

\section{Polymorphism in KITLG, rs4590952, Does not Affect the Risk of Breast Cancer}

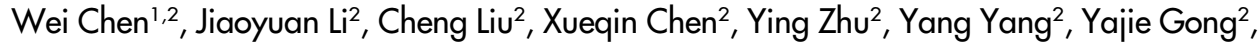 \\ Tengfei Wang ${ }^{2}$, Xiaoping Miao ${ }^{2} \&$ Xiu Nie'
}

${ }^{1}$ Department of Pathology, Union Hospital, Tongii Medical College, Huazhong University of Science and Technology, Wuhan,
China, ${ }^{2}$ State Key Laboratory of Environment Health (Incubation), MOE (Ministry of Education) Key Laboratory of Environment \&
Health, Ministry of Environmental Protection Key Laboratory of Environment and Health (Wuhan), and Department of Epidemiology Health, Ministry of Environmental Protection Key Laboratory of Environment and Health (Wuhan), and Department of Epidemiology
and Biostatistics, School of Public Health, Tongji Medical College, Huazhong University of Science and Technology, Wuhan, China.

Recently, a functional polymorphism in KITLG, rs4590952, was identified to be associated with testicular cancer susceptibility through increasing the p53-dependent KITLG expression and disrupting the function of p53. We performed a hospital-based case-control study, including 1241 breast cancer (BC) patients and 1259 cancer-free controls, to investigate the role of this polymorphism in the risk of BC in Chinese Han population. However, no significant association between rs4590952 and BC risk was identified in allelic model with the odds ratio $(\mathrm{OR})$ of $1.04(95 \%$ confidence interval $(\mathrm{CI})=0.73-1.46, \mathrm{P}=0.839)$ or in any other genetic models. When performed stratified analysis according to the Estrogen Receptor (ER) and Progesterone Receptor (PR) status, rs4590952 was neither associated with ER+/PR+ nor ER-PRsubgroups. Our results suggested that rs4590952 was not associated with the risk of BC in Chinese population, implying that heterogeneous distinct mechanisms might exist in the etiology of different cancers.

ecent genome-wide association studies (GWAS) have revealed numerous low-penetrance common polymorphisms contributing to cancer susceptibility ${ }^{1}$. However, most cancer-associated polymorphisms identified by GWAS are located in noncoding region of genes with the biological mechanisms still not completely clear. In addition, these identified genetic loci can explain only a small part of the cancer susceptibility indicating there may be a substantial fraction of polymorphisms undiscovered, including potentially cancercausing polymorphisms ${ }^{2}$.

Recently, three polymorphisms, rs995030, rs3782181 and rs4474514 have been shown to be associated with differential risk for developing testicular cancer in Caucasians with the per allele odds ratio (OR) of up to 3.07 by GWAS $^{3-5}$. However, the modus operandi of these polymorphisms' function has not been demonstrated. Most recently, a study using genome-wide data sets of cancer susceptibility loci and systematically biological function experiments identified a novel genetic polymorphism, rs4590952, which is located in the p53 response element of KITLG, associated with testicular cancer risk $^{6}$. Intriguingly, this polymorphism was highly correlated to these three polymorphisms, rs995030, rs3782181 and rs4474514. The authors further found that rs4590952 has undergone natural selection throughout evolution with a selective benefit and contributed to testicular cancer susceptibility through disrupting the function of p53.

P53, one of the most famous transcription factors, has been documented to be an important tumor suppressor ${ }^{7}$. The 53 thwarts tumorigenesis by its central monitor role of the cellular stress response pathway ${ }^{8}$. A wide variety of cellular stress signals such as DNA damage, oncogene activation or hypoxia can trigger p53 and then initiate DNA repair, cell cycle arrest, apoptosis and senescence ${ }^{9}$. By monitoring the DNA damage response, p53 acts as a key role in maintaining the integrity of the genome. Furthermore, it has been identified that p53 involved in multiple other biological procedures such as cellular energy metabolism, pigmentation, mitochondrial respiration and stem cell maintenance. Numerous studies indicated that the diverse functions of p53 covers the whole transcriptional, posttranscriptional and posttranslational procedures ${ }^{10}$.

As a transcription factor, p53 mainly exerts its function by interacting with numerous target genes, which potentially contribute to cancer susceptibility ${ }^{8}$. P53 directly binds a DNA consensus site, the p53 response element, of a target gene via its centrally located specific DNA-binding domain (DBD $)^{11}$. Generally, it binds 
the p53 response element as a homo-tetramer and then regulates transcription by a transactivation domain. The ability of p53 to bind the p53 response element and regulate transcription is crucial for p53's tumor suppressor function ${ }^{12}$. Therefore, it was hypothesized that polymorphisms in key nucleotides of functional p53 response elements could influence the ability of p53's transcription regulation and thus might lead to different cancer risk ${ }^{13}$. In consideration of the tumor suppressor role of $\mathrm{p} 53$ in breast cancer $(\mathrm{BC})^{14}$, we hypothesized that rs4590952 might also contribute to susceptibility of BC through affecting the expression of $\mathrm{p} 53$-dependent KITLG and thus influencing the activity of $\mathrm{p} 53$. Hence, we performed a hospital-based case-control study in a Chinese population to test this hypothesis.

\section{Results}

Characteristics of study subjects. The characteristics of the study subjects are presented in Table 1 . The average age between cases and controls was comparable $(P=0.942)$. In addition, there were no significant differences in smoking habit, alcohol use and menopausal status between case and control group. Among the BC subjects, $802(64.60 \%)$ cases were defined as ER positive and 439 (35.40\%) ER negative. Similarly, 712 (57.40\%) cases were PR positive while 529 (42.6\%) were PR negative.

Association analysis. Table 2 describes the genotypes distribution in case and control groups. The genotype of the rs4590952 in controls conformed to Hardy-Weinberg equilibrium (HWE) with a $P$ value of 0.139 . After adjusted by age, smoking habit, alcohol use and menopausal status, the logistic regression analysis showed the A allele was not significantly associated with BC risk, with an OR of $1.04(95 \% \mathrm{CI}=0.73-1.46)$ as compared to the $\mathrm{G}$ allele. No associations were also observed for the heterozygote and homozygote variant, with the ORs of 0.97 (95\% CI $=0.82-1.15$ ) and 1.03 (95\% CI $=0.72-1.46)$, respectively. Additionally, all the dominant, recessive and additive models also showed no association of this polymorphism with BC risk.

When performed stratified analysis by the ER and PR status, no significant associations of rs4590952 and BC risk were found in $\mathrm{ER}+/ \mathrm{PR}+$ and $\mathrm{ER}-\mathrm{PR}-$ subgroups (Table S1).

\section{Discussion}

In this current study, we proposed a hypothesis that rs4590952, which disrupts the function of p53 and undergoes positive selection with selective benefit might contribute to the genetic susceptibility of BC. The SNP rs4590952 was located in KITLG, a common p53 target gene involved in cell growth and differentiation ${ }^{15}$. It was reported that rs4590952 was frequently occupied by p53 in an allele-specific manner and the $G$ allele created a stronger $p 53$ response element with higher affinity when compared to the A allele. In addition, the $G$ allele conferring significantly higher expression of the KITLG than the A allele, indicating the polymorphism may also possessed of enhancer activity. That is to say, the polymorphism of rs4590952 might influence the expression of KITLG and thus disrupting the function of $\mathrm{p} 53$.

Interestingly, in normal mammary gland epithelium the expression of KITLG is significantly higher than that in breast tumor, indicating KITLG might be involved in normal mammary homeostasis and its disruption would confer to breast tumorigenesis ${ }^{16,17}$. However, in this current case-control study including 1241 BC cases and 1259 controls, we did not find evidence of the association between this polymorphism and BC risk under all genetic models. When performed stratified analysis according to the ER and PR status of the BC patients, we still did not find significant associations of rs4590952 and $\mathrm{BC}$ risk in both $\mathrm{ER}+/ \mathrm{PR}+$ and $\mathrm{ER}-\mathrm{PR}-$ subgroups.

The heterogeneous role of rs4590952 in BC and testicular cancer development might be partly due to the different oncogenic properties of p53 and its target genes in different cancers, exemplified by the evidence that $23 \%$ of breast cancers harbor p53 mutations compared to less than $3 \%$ of testicular cancers ${ }^{18}$. This may suggest that the p53 pathway acts differently in different cancers. In addition, the diversity of natural selection pressures and ethnic characteristics among different populations may also contribute to the distinct role of this polymorphism in human cancers.

Although our study firstly investigated the association between rs4590952 and BC risk, some limitations still should be issued. This was a hospital-based case-control study, and thus selection bias was unavoidable and the participants were not being fully representative of the general population. The polymorphism investigated herein, based on its functional consideration, did not offer a comprehensive view of all genetic variants of KITLG. There was also lack of other information on exposures to environmental risk factors. Hence, further studies are needed to investigate the gene-environment interaction effects in BC development.

In conclusion, the current study suggests that the functional polymorphism, rs4590952, does not confer to the risk of BC. The results of our study emphasize the potentially tissue-specific role of this polymorphism in cancer susceptibility. Nevertheless, larger population-based or cohort studies with comprehensive design are needed

\begin{tabular}{|c|c|c|c|}
\hline $\begin{array}{l}\text { Age } \\
\text { Smoking } \\
\text { Yes } \\
\text { No } \\
\text { Alcohol use } \\
\text { Yes } \\
\text { No } \\
\text { Menopausal status } \\
\text { Post-menopause } \\
\text { Pre-menopause } \\
\text { Estrogen Receptor(ER) } \\
\text { Positive } \\
\text { Negative } \\
\text { Progesterone Receptor(PR) } \\
\text { Positive } \\
\text { Negative }\end{array}$ & $\begin{array}{c}49.04 \pm 9.96 \\
9(0.70) \\
1232(99.30) \\
12(1.00) \\
1229(99.00) \\
\\
533(42.90) \\
708(57.10) \\
802(64.60) \\
439(35.40) \\
712(57.40) \\
529(42.60)\end{array}$ & $\begin{array}{c}49.08 \pm 13.75 \\
17(1.40) \\
1242(98.60) \\
14(1.10) \\
1245(98.90) \\
\\
580(46.10) \\
679(53.90)\end{array}$ & $0.721^{b}$ \\
\hline
\end{tabular}


Table 2 | The associations between rs4590952 and BC risk in Chinese population

\begin{tabular}{lcccccc} 
KITLG-rs4590952 & Case (1241) No (\%) & Control (1259) No (\%) & $P^{c}$ & OR $^{c}(95 \% \mathrm{Cl})$ & $P^{\mathrm{b}}$ & $\mathrm{OR}^{\mathrm{a}}(95 \% \mathrm{Cl})$ \\
\hline GG & $778(62.70)$ & $782(62.10)$ & & & & \\
GA & $394(31.70)$ & $409(32.50)$ & 0.711 & $0.97(0.82-1.15)$ & 0.720 & $0.97(0.82-1.15)$ \\
AA & $69(5.60)$ & $68(5.40)$ & 0.912 & $1.02(0.72-1.45)$ & 0.888 & $1.03(0.72-1.46)$ \\
A/G & & & 0.861 & $1.03(0.73-1.46)$ & 0.839 & $1.04(0.73-1.46)$ \\
Dominant & & 0.765 & $0.98(0.83-1.15)$ & 0.781 & $0.98(0.83-1.15)$ \\
Recessive & & 0.861 & $1.03(0.73-1.46)$ & 0.839 & $1.04(0.59-1.46)$ \\
Additive & & & 0.860 & $0.99(0.87-1.13)$ & 0.882 & $0.99(0.87-1.13)$ \\
\hline
\end{tabular}

a: adjusted by age, smoking, alcohol use and menopause status.

b: all statistical tests were two-sided and $P<0.05$ was used as the criterion of statistical significance.

c: calculated without adjustments.

to further clarify the role of polymorphisms in KITLG in genetic susceptibility of BC.

\section{Methods}

Study subjects. This hospital-based case-control study consisted of $1241 \mathrm{BC}$ patients and 1259 cancer-free controls. In this study, with the alpha threshold of 0.05 , the estimated power was $99.74 \%$ or $85.88 \%$ for an odds ratio (OR) of 1.5 or 1.3 , respectively. Patients were pathologically confirmed without restriction in regards of age and histological type and were consecutively recruited from Union Hospital of Huazhong University of Science and Technology, Wuhan, China between June 2009 and Dec 2012. Those who had a history of cancer or metastasized cancer from other organs were excluded from the case group. All of the controls were randomly selected from health examination persons in the same hospital during the same time. These controls were frequency-matched to the cases on age. All subjects were genetically unrelated ethnic Chinese women from Wuhan City and surrounding regions and part of them have been described in elsewhere ${ }^{19-21}$. At recruitment, written informed consent and demographic characteristics along with $2 \mathrm{ml}$ peripheral venous blood were obtained from each subject. The estrogen receptor (ER) and progesterone receptor (PR) status of $\mathrm{BC}$ patients were abstracted from the medical records. Participants who had never smoked or smoked $<1$ year and $<1$ cigarette per day were considered as non-smokers; otherwise, they were considered as smokers. As for the drinking habit, individuals who drank at least twice a week and continued for more than one year were classified as drinkers, otherwise, they were defined as nondrinkers. This study was conducted under the approvable of the review board of Tongji Medical College, Huazhong University of Science and Technology.

Genotyping. Genomic DNA was extracted from the blood samples of each subject using the RelaxGene Blood System DP319-02 (Tiangen, Beijing, China) under the instruction of manufacturer. The genotype of rs 4590952 was determined by the TaqMan SNP Genotyping Assay (Applied Biosystems, Foster city, CA) using the 7900HT Fast Real-Time PCR System (Applied Biosystems, Foster city, CA). All methods were carried out in accordance with the approved guidelines. For quality control, genotyping was performed without the knowledge of participants' status. In addition, $5 \%$ random samples were genotyped twice and the reproducibility was $100 \%$.

Statistical analyses. The difference in distribution of demographic characteristics between cases and controls were assessed by $\chi^{2}$ test or $t$ test, where appropriate. The Hardy-Weinberg equilibrium (HWE) for the genotypes of rs4590952 in control group was evaluated by goodness-of-fit $\chi^{2}$ test. The association between rs4590952 and $\mathrm{BC}$ risk was evaluated by the OR and its $95 \%$ confidence interval (95\% CI) using unconditional logistic regression analysis adjusted by age, smoking habit, alcohol use and menopausal status. All $P$ values were two-tailed with a significance level of 0.05 . The statistical analyses were performed with the SPSS 12.0 software.

1. Hindorff, L. A. et al. Potential etiologic and functional implications of genomewide association loci for human diseases and traits. P Natl Acad Sci USA 106 , 9362-9367 (2009).

2. Michailidou, K. et al. Large-scale genotyping identifies 41 new loci associated with breast cancer risk. Nat Genet 45, 353-361, 361e351-352 (2013).

3. Kanetsky, P. A. et al. Common variation in KITLG and at $5 \mathrm{q} 31.3$ predisposes to testicular germ cell cancer. Nat Genet 41, 811-815 (2009).

4. Rapley, E. A. et al. A genome-wide association study of testicular germ cell tumor. Nat Genet 41, 807-810 (2009).

5. Turnbull, C. et al. Variants near DMRT1, TERT and ATF7IP are associated with testicular germ cell cancer. Nat Genet 42, 604-607 (2010).

6. Zeron-Medina, J. et al. A Polymorphic p53 Response Element in KIT Ligand Influences Cancer Risk and Has Undergone Natural Selection. Cell 155, 410-422 (2013).
7. Junttila, M. R. \& Evan, G. I. p53--a Jack of all trades but master of none. Nat Rev Cancer 9, 821-829 (2009).

8. Vousden, K. H. \& Prives, C. Blinded by the Light: The Growing Complexity of p53. Cell 137, 413-431 (2009).

9. Harris, S. L. \& Levine, A. J. The p53 pathway: positive and negative feedback loops. Oncogene 24, 2899-2908 (2005).

10. Suzuki, K. \& Matsubara, H. Recent advances in $\mathrm{p} 53$ research and cancer treatment. J Biomed Biotechnol 2011, 978312 (2011).

11. Beckerman, R. \& Prives, C. Transcriptional regulation by p53. CSH Perspect Biol 2, a000935 (2010).

12. Crook, T., Marston, N. J., Sara, E. A. \& Vousden, K. H. Transcriptional activation by 553 correlates with suppression of growth but not transformation. Cell 79, 817-827 (1994).

13. Noureddine, M. A. et al. Probing the functional impact of sequence variation on p53-DNA interactions using a novel microsphere assay for protein-DNA binding with human cell extracts. PLoS Genet 5, el000462 (2009).

14. Gasco, M., Shami, S. \& Crook, T. The p53 pathway in breast cancer. Breast Cancer Res 4, 70-76 (2002).

15. Wei, C. L. et al. A global map of p53 transcription-factor binding sites in the human genome. Cell 124, 207-219 (2006).

16. Ulivi, P. et al. c-kit and SCF expression in normal and tumor breast tissue. Breast Cancer Res $\operatorname{Tr}$ 83, 33-42 (2004).

17. Lammie, A. et al. Expression of c-kit and kit ligand proteins in normal human tissues. J Histochem Cytochem 42, 1417-1425 (1994).

18. Walerych, D., Napoli, M., Collavin, L. \& Del Sal, G. The rebel angel: mutant p53 as the driving oncogene in breast cancer. Carcinogenesis 33, 2007-2017 (2012).

19. Chen, W. et al. The SLC4A7 variant rs4973768 is associated with breast cancer risk: evidence from a case-control study and a meta-analysis. Breast Cancer Res $\mathrm{Tr}$ 136, 847-857 (2012).

20. Chen, W. et al. A Functional Variant rs 1820453 in YAP1 and Breast Cancer Risk in Chinese Population. PLoS One 8, e79056 (2013).

21. Chen, W. et al. Risk of GWAS-identified genetic variants for breast cancer in a Chinese population: a multiple interaction analysis. Breast Cancer Res $\operatorname{Tr} \mathbf{1 4 2}$, 637-644 (2013).

\section{Author contributions}

X.P.M. Conceived and designed the study. X.N. and X.P.M. supervised the study. W.C., X.N. and X.P.M. drafted and revised the manuscript. W.C. and J.Y.L. performed the experiments. W.C., C.L. and X.Q.C. conducted statistical analysis. Y.Z., Y.Y., Y.J.G. and T.F.W. participated in the sample collection. All authors reviewed the manuscript.

\section{Additional information}

Supplementary information accompanies this paper at http://www.nature.com/ scientificreports

Competing financial interests: The authors declare no competing financial interests. How to cite this article: Chen, W. et al. A Functional p53 Responsive Polymorphism in KITLG, rs4590952, Does not Affect the Risk of Breast Cancer. Sci. Rep. 4, 6371; DOI:10.1038/srep06371 (2014).

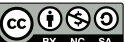

This work is licensed under a Creative Commons Attribution-NonCommercialShareAlike 4.0 International License. The images or other third party material in this article are included in the article's Creative Commons license, unless indicated otherwise in the credit line; if the material is not included under the Creative Commons license, users will need to obtain permission from the license holder in order to reproduce the material. To view a copy of this license, visit http:// creativecommons.org/licenses/by-nc-sa/4.0/ 